\title{
Video Based Behavioral Analysis to Observe Attention during Mirror Therapy in Hemiplegic Patients : Preliminary Study
}

\author{
Jinmin Kim ${ }^{\mathrm{a}}$, Changho Song ${ }^{\mathrm{a}}$ \\ aDepartment of Physical Therapy, College of Health and Welfare, Sahmyook University, Seoul, Republic of Korea
}

\begin{abstract}
Objective: Mirror therapy is one of the promising methods suggested for the upper limb rehabilitation of stroke patients. While mirror therapy was presented to be effective in improving motor function of stroke patients, problems were raised as preceding studies had various methods in applying mirror therapy. Some studies even reported no effect in mirror therapy. Our supposition for the reason of such problems was a decrease of attention on the illusive image during mirror therapy of stroke patients, and we aimed to observe this.
\end{abstract}

Design: A cross-sectional preliminary study.

Methods: Three hemiplegic acute patients were recruited for the traditional mirror therapy. It lasted for 30 minutes, and 12 different tasks were asked to perform. All procedures were video-recorded, and Observer XT was used to analyse mirror-gazing time, gaze-distracted time, preparation time, frequency of mirror gazing, frequency of distraction, frequency of preparation for treatment.

Results: Subjects spent an average of 4-5 minutes having instructions about the mirror therapy intervention (preparation duration), an average of 11 minutes watching the mirror during therapy (mirror-gazing duration), and the rest of 14-15 minutes looking around the environment (gaze-distracted duration). During the mirror therapy, the number of distracted moments (frequency of distraction) was eight times more than focusing on the mirror (frequency of mirror gazing).

Conclusions: Once the patient looks at the mirror, it only lasts about 5 seconds on average. Thus, we confirmed that patients could not concentrate on the illusion during the session and therefore it may have affected the effectiveness of the therapy.

Key Words: Mirror Therapy, Attention, Behavioural analysis

\begin{abstract}
서론
약 15년 전, Ramachandran은 집에서 흔히 사용하는 거울이 치료에 활용되는 것을 제안하였다. 거울 치료는 앉은 자세에서 책상에 손을 올려놓고, 양 팔 사이에 수직 으로 거울을 세운다. 이 때, 거울은 한쪽 팔을 반사하게 되고, 다른 한쪽 팔은 거울 뒤로 가리운다. 치료사는 대상 자에게 거울을 보도록 지시하고, 거울 속의 팔을 거울 뒤 에 있는 진짜 팔처럼 느끼게 하는 환시를 유도한다. 이 중 재는 절단 환자들의 환상 통증을 완화시키는 방법으로 가 장 먼저 소개되었다[1]. 절단 환자들이 거울을 바라볼 때, 절단되었던 팔이 예전처럼 온전하게 있다고 느끼게 되었
\end{abstract}

다. 거울 치료는 환상통을 완화시키는데 효과를 보였고, 이를 바탕으로 뇌졸중 환자에게 적용하는 것이 제안되었 다[2]. 절단 환자들에게 절단 측과 건강한 측이 있는 것처 럼, 뇌졸중 환자 가운데 손상측(마비측)과 건강한 측이 구 별되는 편마비 환자들에게 추천되었다. 뇌졸중의 재활 기 간은 길고, 재활 장비들도 대부분 비싸다는 점에서 환자 와 보호자는 부담을 갖는다. 하지만, 거울 치료는 저렴하 고, 환자의 주도하에 진행할 수 있다는 점에서 많은 사람 들의 관심을 불러일으켰다. 수많은 임상가들은 뇌졸중 환 자에게 꾸준히 이 중재를 적용하여 그 유효성을 증명하고 자 노력해왔다. 이러한 노력은 최근의 코크란 리뷰에서 거울 치료가 뇌졸중 환자의 운동 기능 증진에 효과가 있

Received: Jun 13, 2021 Revised: Jun 15, 2021 Accepted: Jun 16, 2021

Corresponding author: Changho Song (ORCID https://orcid.org/https://orcid.org/0000-0002-5709-3100)

Department of Physical Therapy, College of Health and Welfare, Sahmyook University

Hwarangro 815, Nowon-gu, Seoul, Republic of Korea [01795]

Tel: + 82-2-3213-1123 Fax: +82-2-3399-1638 E-mail: chsong@syu.ac.kr

This is an Open-Access article distributed under the terms of the Creative Commons Attribution Non-Commercial License (http://creativecommons.org/licenses/ by-nc/4.0) which permits unrestricted non-commercial use, distribution, and reproduction in any medium, provided the original work is properly cited.

Copyright @ 2021 Korean Academy of Physical Therapy Rehabilitation Science 
다는 결론을 도출해냈다[3]. 하지만, 거울 치료의 효과가 뚜렷한 차이를 나타냈다고 평가되기 어려우며[4], 심지어 일부 연구에서는 거울 치료가 뇌졸중 환자들의 운동 기능 증진에 효과가 유의미하지 않다고 보고하기도 하였다[5].

거울 치료 효과를 감소시키거나 확인하지 못한 이유 중 하나로 환자가 정해진 치료 시간 가운데 거울을 바라보고 환시를 느끼는 시간의 부족이 원인이 될 수 있다. 뇌졸중 환자의 $46 \% \sim 92 \%$ 에서 주의력 결핍이 나타난다[6]. 그러 므로 뇌졸중 환자의 재활에서 환자의 흥미를 유발하고 목 표하고자 하는 과제를 성취시키도록 유도하는 일은 치료 사의 중요한 역할 중 하나이다. 환자가 거울 속의 상을 보 고 환시를 느껴야 하는 거울치료에서는 환자의 환시에 대 한 집중과 흥미는 중요하다[7]. 환시에 집중하는 것은 거 울 뒤에 있는 손의 운동 기능 효과에 직접적인 영향을 미 칠 수 있다. 그러므로, 환자가 더 환시에 집중할 수 있는 조건을 제공하는 것은 임상적으로 중요한 의미를 제공할 수 있다.

거울 치료시 뇌졸중 환자가 응시하는 곳, 응시하는 기 간 등을 측정하기 위해 Observer XT 소프트웨어는 효과 적인 도구이다. 이 분석 프로그램은 행동을 관찰하여 의 미 있는 코딩 체계 개발, 데이터 입력, 데이터 관리, 데이 터 분석 등을 가능하게 한다. 환자의 움직임을 관찰하는 데 비침습적이고, 접촉없이 비디오 촬영만을 통해 가능하 다. 특히 운동역학적인 평가가 어려운 부위의 움직임 또 한 분석이 가능하도록 하여, 움직임의 질을 평가하는 실 험에 활용이 적합하다[8].

따라서 본 연구는 예비실험으로 행동 분석 프로그램을 통하여 뇌졸중 환자가 거울 치료시 응시하는 곳과 기간, 빈도 등을 조사하여 환자가 거울 치료 동안 집중이 얼마 나 이루어지는지 확인하고자 한다.

\section{연구 방법}

\section{연구 대상}

본 연구는 경기도에 소재한 $\mathrm{O}$ 병원에 내원하고 있는 환자 3 명을 대상으로 하였으며, 연구의 목적과 방법을 내 원하고 있는 환자들에게 홍보하여 모집하였다. 연구대상 자의 선정 조건은 뇌졸중으로 인한 편마비 환자로 유병 기간이 12 개월 이내이며 간단한 말을 이해하여 의사소통 이 가능한 자, 한국형 간이상태정신검사 점수가 21점 이 상이 자, Fugl-meyer score가 26-56점 사이로 경하거나 중간 정도의 운동 손상이 있는 자이었다. 제외 조건은 치 매나 다른 정신과적인 문제가 있는 자, 편측 무시나 실행 증이있는 자, 상지의 다른 정형외과적 문제가 있는 자이 었다. 홍보를 통해 모집된 환자 중 선정 조건에 부합하고
연구에 대한 충분한 이해를 하고 동의한 자로 하였다. 모 든 연구자는 자발적으로 동의서에 서명을 하였으며 본 연 구는 삼육대학교의 연구윤리위원회의 승인을 얻어 시행 하였다.

\section{연구 절차}

모든 대상자에게 거울치료를 각기 다른 날에 시행하였 으며 치료시간은 30 분이며 대상자 마다 3 회의 치료를 시 행하였다. 치료시 촬영된 자료는 행동분석 소프트웨어에 의해 분석되었으며[8], 치료에 집중하는 시간을 확인하기 위해 거울 응시하는 시간과 빈도로 나누어 분석하였다. 치료시간을 응시시간과 준비시간으로 나누고 응시시간은 거울을 응시하는 시간과 주변을 응시하는 시간으로 나누 었다. 거울을 응시하는 시간을 치료에 주의하는 시간으로 정의하였다. 응시하는 시간을 빈도로 나누어 평균 1 회 응 시시간을 구하였다.

\section{중재 방법}

\section{거울치료}

거울치료는 가로세로 $30 \mathrm{~cm} \times 30 \mathrm{~cm}$ 크기의 거울로 시 행하였다. 환자는 의자나 휠체어에 앉고, 거울은 환자 앞 테이블에 수직으로 놓았다. 환자의 건측팔을 거울 앞쪽에 놓고 환측팔을 거울 뒤에 놓아 거울에 반사된 건측팔이 환측 팔처럼 보이도록 환시를 유도하였다. 거울치료프로 그램은 과제는 11 개로 구성되었다. 테이블 닦기, 팔을 앞 으로 밀기, 테이블을 따라 물체이동, 물체뒤집기, 컵 들었 다 놓기, 다양한 크기의 물체잡기, 페이지 넘기기, 동전을 저금통의 구멍에 넣기, 바둑알을 손바닥에 올리기, 클립을 집기, 구멍에 못을 꽂기.

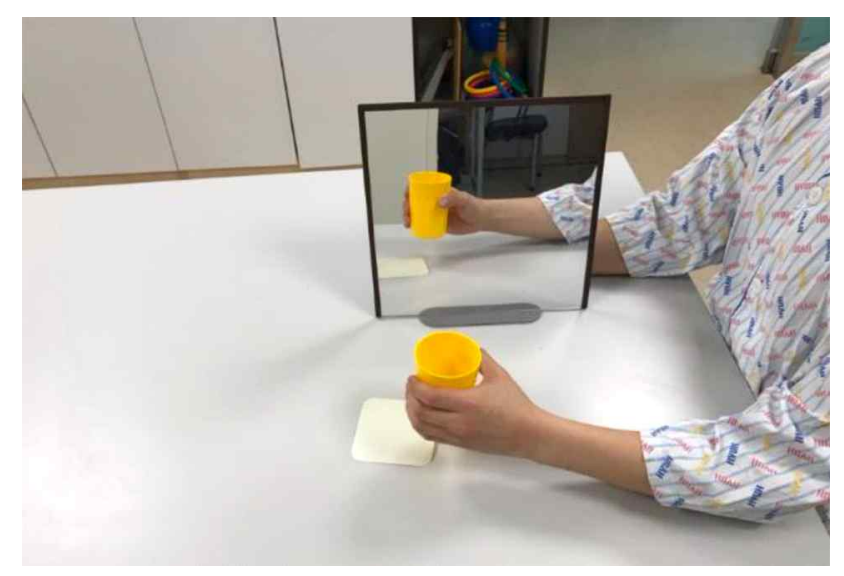

Figure 1. Reaching and Grasping a Cup during Mirror Therapy 


\section{측정방법 및 도구}

본 연구의 행동 분석을 위해 행동 분석 소프트웨어 Observer XT를 사용하였다. Observer XT는 코딩 체계 개발, 데이터 입력, 데이터 관리, 데이터 분석, 그리고 상 호 신뢰도 분석을 위해 사용되는 행동 코딩 및 분석 소프 트웨어이다. 캠코더로 녹화된 비디오 파일을 Observer $\mathrm{XT}$ 프로그램 불러와 연구자가 미리 계획한 코딩 체계를 통해 코딩을 하게 된다. 본 연구에서는 치료시의 환자의 응시를 분석하였다. 분석은 숙련된 연구자에 의해 진행하 였다. 거울을 응시하는 상황을 코딩하였고 Observer XT 에 의해 응시 시간과 빈도를 분석하였다.

\section{연구 결과}

30 분의 치료시간 중 치료 시간 중 거울응시시간은 평균 649.36초, 거울이 아닌 다른 곳을 응시한 시간은 평균 871.24초, 과제를 준비하는 데 소모된 시간은 평균 279.40 초이었다. 전체 시간의 백분율로 나타내면, 거울응 시시간은 약 $36 \%$, 다른 곳을 응시한 시간은 약 $48 \%$, 과 제 준비를 위해 소모된 시간은 전체 치료시간의 약 $16 \%$ 를 차지했다.

치료시간 중, 거울을 쳐다본 빈도 수는 124.32회였고, 다른 곳을 바라본 빈도수는 137.93회였다. 한번 거울을 바라볼 때 거울을 얼마나 오래동안 바라보고 있는가를 확 인하기 위해서는 거울응시시간을 거울을 쳐다본 빈도수로 나누어 확인하였다. 이 때, 평균 5.28초 바라보는 것으로 나타났다.
Table 1. General Characteristics of Participants $\quad(n=3)$

\begin{tabular}{llll}
\hline Characteristics & Subject 1 & Subject 2 & Subject 3 \\
\hline Sex & male & male & female \\
Age (years) & 62 & 57 & 59 \\
Height $(\mathrm{cm})$ & 172 & 169 & 154 \\
Weight $(\mathrm{kg})$ & 64 & 72 & 52 \\
Onset time & 7 & 4 & 6 \\
(month) & & & \\
Stroke type & infarction & infarction & infarction \\
Paretic side & left & right & right \\
MMSE-K & 24 & 25 & 26 \\
\hline
\end{tabular}

Mirror therapy intervention

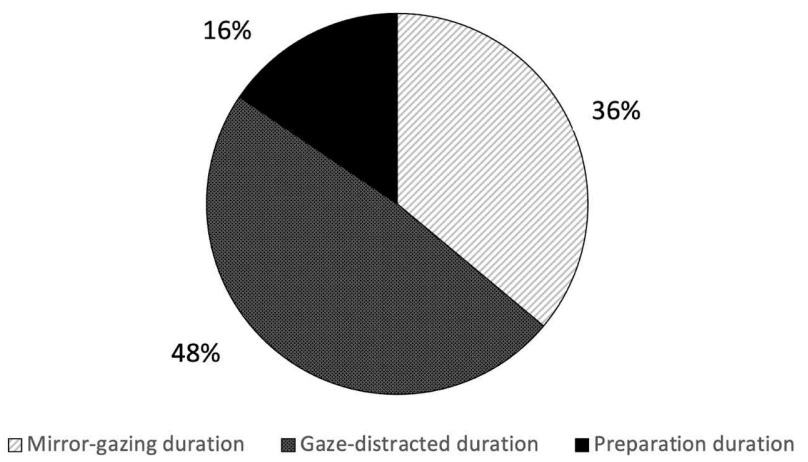

Figure 2. Mirror Therapy Gaze Duration

Table 2. The Gaze Duration and Frequency of Participants

$(n=3)$

\begin{tabular}{|c|c|c|c|c|c|c|c|c|}
\hline \multirow[b]{2}{*}{$\begin{array}{l}\text { Mirror gazing duration } \\
(\mathrm{sec})\end{array}$} & \multicolumn{2}{|c|}{ Subject 1} & \multicolumn{2}{|c|}{ Subject 2} & \multicolumn{2}{|c|}{ Subject 3} & \multicolumn{2}{|l|}{ Total } \\
\hline & 715.16 & $(75.98)$ & 630.72 & $(105.57)$ & 602.20 & $(162.05)$ & 649.36 & (115.69) \\
\hline $\begin{array}{l}\text { Gaze-distracted duration } \\
(\mathrm{sec})\end{array}$ & 947.58 & (139.09) & 805.67 & (189.97) & 860.46 & (109.65) & 871.24 & (143.89) \\
\hline $\begin{array}{l}\text { Preparation duration } \\
(\mathrm{sec})\end{array}$ & 137.25 & (17.26) & 363.61 & $(24.65)$ & 337.34 & $(24.32)$ & 279.40 & (29.76) \\
\hline $\begin{array}{l}\text { Mirror gazing frequency } \\
\text { (n) }\end{array}$ & 130.88 & (13.81) & 132.40 & $(9.80)$ & 109.69 & $(8.03)$ & 124.32 & (14.45) \\
\hline $\begin{array}{l}\text { Gaze-distracted frequency } \\
\text { (n) }\end{array}$ & 135.57 & (4.20) & 142.44 & (11.75) & 135.77 & $(9.82)$ & 137.93 & $(8.63)$ \\
\hline $\begin{array}{l}\text { Preparation frequency } \\
\text { (n) }\end{array}$ & 16.28 & (2.72) & 15.23 & $(0.76)$ & 14.77 & $(1.21)$ & 15.43 & $(1.67)$ \\
\hline $\begin{array}{l}\text { Average duration } 1 \text { time } \\
(\mathrm{sec})\end{array}$ & 5.49 & $(0.66)$ & 4.81 & $(1.12)$ & 5.53 & $(1.59)$ & 5.28 & $(1.08)$ \\
\hline
\end{tabular}

The values are presented mean (SD) 


\section{고찰}

본 연구는 행동 분석 프로그램을 통하여 뇌졸중 환자가 거울 치료시 응시하는 곳과 기간, 빈도 등을 조사하여 환 자가 거울 치료 동안 집중이 얼마나 이루어지는지 확인하 는 예비 연구로 다음과 같은 결과를 얻었다. 전체 치료 시 간 중 $16 \%$ 는 치료를 준비하는 시간이었으며 실제 치료 시간은 $84 \%$ 였다. 이 중 거울에 집중하는 시간은 30 분의 치료 시간 중 10.82 분으로 $36 \%$ 에 해당하였다. 주변을 응 시하는 시간은 14.52 분으로 $48 \%$ 에 해당하였다. 뇌졸중 환자가 거울 치료를 받을 때 치료에 집중하는 시간 보다 집중하지 못하는 시간이 더 큰 것으로 나타났다. 빈도 분 석을 통해 평균 1 회 응시지속시간을 구해보면 5.28초로 매우 짧은 시간 동안만 집중하는 것으로 나타났다. 주위 를 응시하는 지속시간은 6.36초로 오히려 더 긴 시간 동 안 집중을 못하는 것으로 나타났다. 거울 치료는 건측의 움직임을 환측의 움직임으로 착각하게 하는 환시를 형성 하여 뇌에 시각정보를 전달하는 것이 치료의 주된 원리로 제안되어 왔다[9]. 환자가 거울에 집중하여 건측의 움직임 을 보고 환시가 발생하기 까지는 일정한 시간이 필요하다. 그러한 시간을 감안한다면 1 회 응시 지속시간인 5.28초에 서 실제 치료의 효과를 보는 시간은 매우 적다고 할 수 있다. 임상가들은 거울치료시 적절한 큐잉과 환경을 조성 하여 치료시 거울에 집중하는 시간을 늘려야한다. 뇌졸중 환자가 거울에 집중하지 못하는 원인을 아는 것이 중요하 다. 본 연구에 참여한 대상자들은 편측무시가 없는 환자 들이었기 때문에 편측무시로 인한 신경학적인 원인이 주 된 원인은 아니었다. 주의력 결핍은 뇌졸중 환자에게 나 타나는 일반적인 장애로 선행 연구에서는 뇌졸중 환자의 46\% 92\%에서 주의력 결핍이 나타난다고 보고하고 있 고 대체적으로 $80 \%$ 이상의 환자에게서 나타난다고 보고 되고 있다 $[6,10]$. 주의력이란 주어진 과제에 초점을 맞추 는 것으로 일반적으로 집중력(concentraion)이라고 부른 다. 선택적 주의는 의도적으로 다른 정보 소스들에 관심 을 피하거나 억제하면서 하나의 정보에 주의를 기울이는 것을 말한다[11]. 분리 주위는 여러 자극을 동시에 통합하 는 능력이다. 분리 주의의 기능은 하나 이상의 활동에서 동시에 주의를 기울이는 능력이다[12]. 뇌졸중 환자의 상 지재활의 여러 방법 중 거울치료는 환시의 과정이 필요하 기 때문에 어느 정도 거울에 집중을 하는 주의력이 필요 하다. 그러나 주의력의 장애는 집중력 감소와 산만함에 원인이 되므로[13], 뇌졸중 환자가 치료에 집중하는 것을 어렵게 한다.

여러 임상연구들에서 거울치료에 대한 임상적 효과를 보고하고 있지만 본 연구의 결과를 토대로 유추해볼 때 과연 치료 중에 실제로 환시를 일으킬 수 있는 시간이 확
보되고 있는가에 대하여 의문이 남게 된다. 본 연구는 3 명의 환자를 대상으로 한 연구로 일반화에는 한계가 있다. 다만 예비연구로서 향후 좀더 많은 표본과 더 다양한 행 동 분석을 하는 연구가 필요하다고 생각한다. 또한 임상 가들은 어떠한 치료를 할 것인가 에만 집중할 것이 아니 라 어떻게 하면 효과적인 거울 치료가 될 것인가에 대한 해답을 얻기 위한 노력이 필요하다.

본 연구는 예비실험으로 참가자의 수가 적어 그 유효성 을 검증하기에 한계가 있다. 그러므로, 더 많은 수의 대상 자를 바탕으로 한 연구가 필요하다. 더불어, 뇌졸중 환자 의 상태에 따라 치료의 집중도가 달라지는 바, 어떠한 지 표가 치료의 집중도에 영향을 미치는지를 살펴볼 필요가 있다.

\section{결론}

본 연구를 통해 환자는 거울치료시 거울에 평균 5 초 정도 집중하는 것으로 나타나 치료의 효과에 영향을 줄 수 있는 가능성이 있는 것으로 확인되었다. 거울치료가 제공하는 환시의 효과를 더 누리도록 하기 위한 개선된 방법이 제시될 필요가 있다.

\section{감사의 글}

이 논문은 2018년도 정부(교육부)의 재원으로 한국연 구재단의 지원을 받아 수행된 기초연구사업임 (No. NRF-2018R1D1A1B07043319)

본 연구의 저자들은 연구, 저자권, 및 출판과 관련하여 잠재적인 이해충돌이 없음을 선언합니다.

\section{참고문헌}

1. Ramachandran VS, Rogers-Ramachandran D, Cobb S. Touching the phantom limb. Nature. 1995;377:489-90.

2. Ramachandran VS, Rogers-Ramachandran D. Mirror feedback assisted recovery from hemiparesis following stroke. In Reply to Morkisch et al.: How to perform mirror therapy after stroke? Evidence from a meta-analysis. Restor Neurol Neurosci. 2019;37:437-43.

3. Thieme H, Morkisch N, Mehrholz J, Pohl M, Behrens J, Borgetto B, et al. Mirror therapy for improving motor function after stroke. Cochrane Database Syst Rev. 2018;7:CD008449.

4. Morkisch N, Thieme H, Dohle C. How to perform mirror therapy after stroke? Evidence from a 
meta-analysis. Restor Neurol Neurosci. 2019;37:421-35.

5. Antoniotti P, Veronelli L, Caronni A, Monti A, Aristidou E, Montesano $\mathrm{M}$, et al. No evidence of effectiveness of mirror therapy early after stroke: an assessor-blinded randomized controlled trial. Clin Rehabil. 2019;33:885-93.

6. Barker-Collo SL, Feigin VL, Lawes CM, Parag V, Senior H, Rodgers A. Reducing attention deficits after stroke using attention process training: a randomized controlled trial. Stroke. 2009;40:3293-8.

7. Kal E, van den Brink H, Houdijk H, van der Kamp J, Goossens PH, van Bennekom C, et al. How physical therapists instruct patients with stroke: an observational study on attentional focus during gait rehabilitation after stroke. DisabilRehabil. 2018;40:1154-65.

8. Ducharme DA, Arcand I. Using Noldus Observer XT for research on deaf signers learning to read: an innovative methodology. Behav Res Methods. 2009;41:833-40.

9. Deconinck FJ, Smorenburg AR, Benham A, Ledebt A, Feltham MG, Savelsbergh GJ. Reflections on mirror therapy: a systematic review of the effect of mirror visual feedback on the brain. Neurorehabil Neural Repair. 2015;29:349-61.

10. Hyndman D, Pickering RM, Ashburn A. The influence of attention deficits on functional recovery post stroke during the first 12 months after discharge from hospital. J Neurol Neurosurg Psychiatry. 2008;79:656-63.

11. Schmidt RA, Lee TD. Motor control and learning: A behavioral emphasis. 5th ed. Champaign: Human Kinetics; 2011.

12. McDowd JM, Craik FIM. Effects of aging and task difficulty on divided attention performance. J Exp Psychol Hum Percept Perform. 1988;14:267-80.

13. Loetscher T, Lincoln NB. Cognitive rehabilitation for attention deficits following stroke. Cochrane Database Syst Rev. 2013:CD002842. 